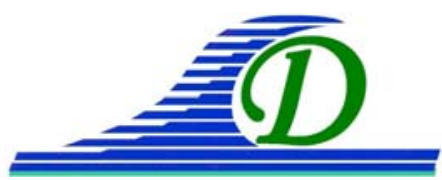

XIII ${ }^{\text {èmes }}$ Journées Nationales Génie Côtier - Génie Civil

Dunkerque, 2-4 juillet 2014

DOI:10.5150/jngcgc.2014.054 C Editions Paralia CFL

disponible en ligne - http://www.paralia.fr - available online

\title{
Distribution non stationnaire des matières en suspension dans une couche limite oscillant à haute fréquence
}

\author{
Martin SANCHEZ ${ }^{1}$
}

1. Université de Nantes, Faculté des Sciences et Techniques,

UMR-6112 du CNRS - Planétologie et Géodynamique,

2 rue de la Houssinière, BP 92208, 44322 Nantes cedex 3, France.

martin.sanchez@univ-nantes.fr

\section{Résumé :}

La validité d'un modèle phénoménologique dénommé $\alpha$ - $\beta$ initialement développé pour décrire la distribution verticale des sédiments fins en suspension (SANCHEZ, 2013) est ici examinée dans le cas de sables et de graviers soumis à des forçages hydrodynamiques cycliques dans une gamme de périodes couvrant des phénomènes très différents tels que la marée et la houle. Les résultats de ce modèle sont comparés avec ceux issus de la résolution numérique d'un deuxième modèle basé sur l'équation théorique 1DV régissant la variation dans le temps de la concentration en matières en suspension. Dans les deux modèles les échanges solides avec le fond sont paramétrés à l'aide d'une fonction "pick-up" d'usage répandu. Les résultats obtenus montrent que le domaine d'application du modèle $\alpha$ - $\beta$ est très large et couvre pratiquement tout type de sédiment transporté en suspension dans les environnements côtiers.

Most-clés : Transport de sédiments en suspension, Distribution verticale de la concentration, Régime non stationnaire, Dépôt, Erosion, Vase, Sable, Gravier.

\section{Introduction}

Les modèles hydrodynamiques 2DH actuels utilisés en milieu océanique et côtier, permettent des applications sur des longues durées, à une échelle géographique fine couvrant de grandes étendues. Si les variations verticales des variables étudiées sont convenablement décrites par une fonction théorique ou semi-théorique, les résultats obtenus sont d'une qualité excellente. Deux applications réussies suivant cette démarche sont :

- les modèles de propagation de la houle sur des fonds peu inclinés qui décrivent la distribution verticale du potentiel de vitesses avec la théorie de Stokes-Airy,

- les modèles courantologiques de fluides homogènes qui intègrent sur toute la profondeur l'effet des contraintes de cisaillement sur les vitesses moyennes.

Un modèle dénommé $\alpha$ - $\beta$ a été récemment développé (SANCHEZ, 2013) dans le but d'inclure, dans les modèles $2 \mathrm{DH}$ de transport des matières en suspension, une description précise de la répartition verticale des sédiments. Quand les échanges solides avec le fond sont paramétrés par un taux de dépôt ou par un taux d'érosion, ce modèle 


\section{Thème 2 - Dynamique sédimentaire}

peut s'appliquer en régime non stationnaire et non uniforme, et ce, bien au-delà de la loi de Rouse-Vanoni qui est valide uniquement pour un régime uniforme et stationnaire. Les validations préalables du modèle $\alpha-\beta$ ont été effectuées pour des vitesses de chute $W$ faibles $\left(0,05<W\left(\mathrm{~mm} \mathrm{~s}^{-1}\right)<3,2\right)$ caractéristiques des sédiments fins et des variations des paramètres hydrodynamiques correspondant à des marées semi-diurnes.

L'objet de cette étude est d'explorer la validité du modèle $\alpha-\beta$ dans une couche limite avec des vitesses de cisaillement oscillant à haute fréquence, couvrant des phénomènes tels que la marée, les surcotes, les seiches et les houles. Les vitesses de chute étudiées correspondent à des sables et des graviers (masse volumique retenue $\rho_{s}=2600 \mathrm{~kg} \mathrm{~m}^{-3}$ ) de diamètre compris entre $0,2 \mathrm{~mm}$ et $10 \mathrm{~mm}$.

\section{Théorie stationnaire}

Si le régime sédimentaire est uniforme et stationnaire, la distribution verticale de la concentration $C$ en matières en suspension est régie par :

$W C=-K_{z} \frac{\partial C}{\partial z}$

où $W$ est la valeur locale de la vitesse de chute des matières en suspension, $z$ la coordonnée verticale et $K_{z}$ le coefficient de diffusion turbulente de masse selon la direction $O z$. Dans ce qui suit on considère que le fond se situe en $z=0$.

L'intégration de l'équation 1 permet d'obtenir la loi de Rouse-Vanoni généralisée pour la distribution verticale de la concentration $C$, qui en en régime stationnaire est ici notée $\dddot{C}$ (SANCHEZ et al., 2005) :

$\dddot{C}(z)=C(0) \exp \left(-\int_{0}^{z} \frac{W}{K_{z}} \mathrm{~d} z\right)$

L'équation précédente peut admettre une solution analytique dépendant des expressions de $K_{z}$ et de $W$. Dans cette étude, ces deux grandeurs sont considérées être indépendantes de $z$. Cette hypothèse est une simplification du problème très utilisée dans les applications pratiques, mais la méthodologie développée permet d'inclure une variabilité verticale de $K_{z}$ et de $W$ comme il est indiqué dans l'annexe 1 de la référence SANCHEZ (2013). L'expression 2 de la concentration devient alors :

$\dddot{C}\left(z^{\circ}\right)=C(0) \exp \left(-\alpha_{\infty} z^{\circ}\right)$

où $z^{\circ}=z / d$ est la coordonnée verticale adimensionnelle, $d$ la profondeur et $\alpha_{\infty}$ le nombre de Peclet caractéristique des transferts verticaux de sédiments par convection-diffusion :

$\alpha_{\infty}=\frac{W d}{K_{z}}$

Une relation usuelle pour évaluer $K_{z}$ qui est retenue dans cette étude est la suivante :

$K_{z}=\frac{\kappa}{6} U_{c} d$

où $U_{c}$ est la vitesse de cisaillement et $\kappa \approx 0,4$ la constante universelle de Karman. 


\section{XIII $I^{\text {emes }}$ Journées Nationales Génie Côtier - Génie Civil \\ Dunkerque, 2-4 juillet 2014}

\section{Présentation du modèle $\alpha-\beta$ (SANCHEZ, 2013)}

Des tests numériques effectués pendant la phase de validation du modèle $\alpha-\beta$ en admettant une absence des phénomènes d'érosion et de dépôt, ont montré qu'avec des conditions hydrodynamiques variables dans le temps, la concentration $C$ n'est plus décrite par l'équation 3. Selon le modèle $\alpha-\beta$, dans ce cas de régime non stationnaire le profil de la concentration noté $\widetilde{C}$ est correctement modélisé par l'équation suivante :

$\widetilde{C}\left(z^{\circ}\right)=C(0) \exp \left(-\alpha z^{\circ}\right)$

où $\alpha$ est un paramètre dont la valeur tend toujours vers $\alpha_{\infty}$ selon une loi phénoménologique qui, en régime uniforme, s'écrit :

$\frac{\partial \alpha}{\partial t}=c_{\alpha} \frac{U_{c}}{d}\left(\alpha_{\infty}-\alpha\right)$

où $c_{0} \approx 0,667$ est le coefficient du modèle.

Le dépôt et l'érosion des sédiments fins modifient de façon plus significative le profil vertical de la concentration $C$ que le caractère non stationnaire des conditions hydrodynamiques. Il est montré que dans tous les cas le profil de $C$ est bien décrit par (SANCHEZ, 2013) :

$C\left(z^{\circ}\right)=C_{R} \exp \left(-\alpha z^{\circ}\right) \exp \left(-\beta\left(1-z^{\circ}\right)^{2}\right)$

où $C_{R}$ est une concentration de référence et $\beta$ un paramètre dont la valeur tend toujours vers $\beta_{\infty}$ selon la loi phénoménologique suivante incluant un coefficient $c_{\beta}$ :

$\frac{\partial \beta}{\partial t}=c_{\beta} \frac{U_{c}}{d}\left(\beta_{\infty}-\beta\right)$

où $\beta_{\infty}$ est la valeur terminale instantanée du paramètre $\beta$, qui dépend des échanges solides entre le fond et la colonne d'eau, échanges paramétrés par le taux de dépôt $D^{e f}$ (terme puits en $\mathrm{kg} \mathrm{m}^{-2} \mathrm{~s}^{-1}$ ) ou le taux d'érosion $E^{e f}$ (terme source en $\mathrm{kg} \mathrm{m}^{-2} \mathrm{~s}^{-1}$ ).

Dans ce qui suit on utilise un seul taux d'échanges solides avec le fond $S^{e f}$ qui est égal à $D^{e f}$ en cas de dépôt et égal à $-E^{e f}$ en cas d'érosion. De même, on définit un taux adimensionnel d'échanges solides avec le fond comme suit :

$p=\frac{S^{e f}}{W \times C(0)}$

Il est montré (SANCHEZ, 2013) que si le paramètre $p$ et les conditions hydrodynamiques restent constantes dans le temps, $\alpha$ converge vers $\alpha_{\infty}$ et $\beta$ vers $\beta_{\infty}$. Dans ce cas les conditions de frontière liées aux échanges solides avec le fond sont satisfaites si :

$\beta_{\infty}=0,50 p \alpha$

En complément, en cas d'érosion ou d'échanges solides nuls avec le fond $(p \leq 0)$ on a :

$c_{\beta}=0,667$

Finalement, en cas de dépôt $(p>0)$ on a :

$c_{\beta}=0,667+0,3 \times \beta_{\infty}$ 


\section{Méthodes}

Un modèle numérique 1DV a été mis au point pour simuler la distribution verticale des matières en suspension. Celui-ci est régi par l'équation théorique suivante :

$$
\frac{\partial C}{\partial t}=\frac{\partial}{\partial z}\left(K_{z} \frac{\partial C}{\partial z}+W C\right)
$$

Des simulations numériques ont été effectuées avec une large gamme de valeurs des variables du problème couvrant un grand nombre de cas réels de transport en suspension de sables et de graviers.

Les simulations ont été effectuées avec une vitesse de cisaillement cyclique donnée par l'expression suivante :

$$
U_{c}=U_{c-\text { moy }}[1-A m p l \times \cos (\omega t)]
$$

où $U_{c-m o y}$ est la vitesse de cisaillement moyenne du test, $A m p l$ un paramètre (dont la valeur est ici fixée à 0,80 ) définissant l'importance des oscillations de la valeur de $U_{c}$ et $\omega=2 \pi / T$ la pulsation qui est reliée à la période $T$ des oscillations.

Les échanges solides avec le fond sont calculés par :

$S^{e f}=W \times C(0)-E$

où $E$ est le taux de remise en suspension évalué à travers la fonction "pick-up" de VAN RIJN (1984). Selon cette fonction, si $U_{c} \leq U_{c-c r i t}, E=0$, et si $U_{c}>U_{c-c r i t}$, on a :

$$
E=0,00033 \rho_{s}\left(\frac{\rho_{s}}{\rho}-1\right)^{0,6} \frac{g^{0,6} D^{0,8}}{v^{0,2}}\left(\frac{U_{c}{ }^{2}-U_{c-c r i t}{ }^{2}}{U_{c-c r i t}{ }^{2}}\right)^{1,5}
$$

où $g=9,81 \mathrm{~m} \mathrm{~s}^{-2}$ est l'accélération de la pesanteur, $v=1 \times 10^{-6} \mathrm{~m}^{2} \mathrm{~s}^{-1}$ la viscosité cinématique de l'eau, $\rho=1000 \mathrm{~kg} \mathrm{~m}^{-3}$ la masse volumique de l'eau, $D$ le diamètre des grains de sédiment et $U_{c-c r i t}$ la valeur de $U_{c}$ critique de remise en suspension. Dans cette étude on a retenu $U_{c \text {-crit }}=W$, ce qui se justifie par le fait que pour $W / U_{c}>\sim 1$ les sédiments non cohésifs ne sont pas transportés en suspension (TEETER, 1986).

Lors des simulations on a fixé $U_{c-m o y}=U_{c-c r i t}$. On a ensuite combiné différentes valeurs pour les variables du problème. Parmi les valeurs étudiées on peut citer :

- Quatre valeurs de $d$ (en m) $: 0,5 ; 1 ; 2$ et 4 .

- Cinq valeurs de $T$ (en s) $: 2,5 ; 5 ; 10 ; 600$ et 22400 .

- Six valeurs de $D$ (en mm) : 0,$2 ; 0,5 ; 1 ; 2 ; 5$ et 10 .

Le tableau 1 donne les vitesses de chute $W$ associées aux valeurs de $D$ étudiées.

Tableau 1. Valeurs de la vitesse de chute reliées aux diamètres des grains de sédiment.

\begin{tabular}{ll}
\hline$D(m)$ & $W\left(\mathrm{~m} \mathrm{~s}^{-1}\right)$ \\
\hline 0,0002 & 0,0264 \\
0,0005 & 0,0723 \\
0,001 & 0,148 \\
0,002 & 0,276 \\
0,005 & 0,488 \\
0,010 & 0,690 \\
\hline
\end{tabular}




\section{XIII ${ }^{\text {èmes }}$ Journées Nationales Génie Côtier - Génie Civil \\ Dunkerque, 2-4 juillet 2014}

Le modèle numérique 1DV décompose la colonne d'eau en $N$ couches d'épaisseur adimensionnelle constante égale à $\Delta z^{\circ}=\Delta z / d=1 / N$. Dans tous les tests $N$ a été fixé à 50 . A chaque instant $t$ de la modélisation, on connait la concentration $C_{i}$ au milieu de chaque couche $i$ de la colonne d'eau dont la cote adimensionnelle par rapport au fond est $z^{\circ}=(i-1 / 2) \Delta z^{\circ}$.

Les mêmes simulations ont été effectuées avec un modèle 1DV et avec un modèle $\alpha-\beta$ complètement indépendant du premier, et ce, afin de valider les résultats du second dans les gammes de périodes et de vitesses de chute ici étudiées.

\section{Résultats}

En accord avec l'équation 15, dans les simulations effectuées avec les modèles 1DV et $\alpha-\beta$, la valeur initiale de $U_{c}$ (à $\left.t=0\right)$ est $U_{c-\text {-initial }}=U_{c-\text { moy }} \times(1-A m p l)$. Selon les équations 4 et 5 la valeur initiale du nombre de Peclet est alors $\alpha_{\infty}=(6 \times W) /\left(\kappa \times U_{\text {c-initial }}\right)$.

Pour le modèle $\alpha-\beta$ les valeurs initiales des paramètres ont été fixées dans toutes les simulations comme suit : $\alpha=0,20 \times \alpha_{\infty}$ et $\beta=0$. Ces valeurs initiales ont un effet sur les résultats qui disparait au bout d'un certain temps. Dans tous les cas ici étudiés on a constaté que les résultats étaient indépendants des conditions initiales pour $t>5 T$, raison pour laquelle, les résultats montrés sur les figures 1 à 5 correspondent à un intervalle de temps défini par $5 T<t<10 T$, intervalle décrivant 5 cycles de variation de $U_{c}$.

Chacune des figures 1 à 5 comporte 3 graphiques :

- Le graphique (a) décrivant la variation des paramètres $\alpha_{\infty}, \alpha, \beta_{\infty}$ et $\beta$.

- Le graphique (b) montrant l'évolution dans le temps de la concentration moyenne sur la verticale $\bar{C}$ d'après les résultats (i) du modèle $1 \mathrm{DV}$, (ii) du modèle $\alpha-\beta$ et (iii) du propre modèle $\alpha-\beta$ quand la valeur de $\beta$ est fixée à zéro (ce dernier cas correspond à un profil de $C$ non modifié par les processus de dépôt et d'érosion).

- Le graphique $(c)$ où le rapport de la concentration au fond sur la concentration moyenne sur la verticale $C(0) / \bar{C}$, est montré d'après ces mêmes résultats décrits cidessus.

On doit signaler qu'une bonne description du rapport $C(0) / \bar{C}$ est la clé pour obtenir des bons résultats dans les modélisations $2 \mathrm{DH}$ du transport des matières en suspension (TEETER, 1986).

\subsection{Résultats pour les fortes périodes ( $T=600 \mathrm{~s}$ et $T=22400 \mathrm{~s}$ )}

Les résultats présentés sur les figures 1 et 2 sont associés à une profondeur $d=2 \mathrm{~m}$ et à un diamètre des grains de sédiment $D=0,5 \mathrm{~mm}$ (vitesse de chute $W=0,0723 \mathrm{~m} \mathrm{~s}^{-1}$ ).

La figure 1 montre les résultats associés à un signal $U_{c}$ d'une période $T=22400 \mathrm{~s}$ imposé dans les simulations numériques. Ce signal est représentatif des ondes de marée semidiurne d'une période de $44800 \mathrm{~s}$. C'est pour des valeurs de $T$ de cet ordre de grandeur que les coefficients $c_{\alpha}$ et $c_{\beta}$ du modèle $\alpha$ - $\beta$ ont été déterminés par SANCHEZ (2013) pour des sédiments fins. 


\section{Thème 2 - Dynamique sédimentaire}

La figure 1(a) montre que $\alpha \approx \alpha_{\infty}$ et que $\beta$ vaut presque toujours zéro, sauf à la fin du processus de dépôt (quand la valeur $\bar{C}$ est en train de se rapprocher de zéro). Selon ces résultats le régime est quasi stationnaire de sorte que le profil vertical de la concentration pourrait être calculé avec la loi de Rouse-Vanoni (équations 2 ou 3).

Les résultats de la figure 1(c), confirment la validité de la loi de Rouse-Vanoni dans ce cas étudié. En effet, les valeurs de $C(0) / \bar{C}$ correspondant aux deux modèles étudiés sont presque identiques et il est de même quand $\beta=0$ ce qui implique que le profil vertical de la concentration n'est pas modifié de façon significative par les processus de dépôt et d'érosion. On doit signaler que le rapport $C(0) / \bar{C}$ correspondant au modèle 1DV ne peut pas être calculé quand $\bar{C} \rightarrow 0$. Dans cette étude ce rapport n'est pas évalué quand $\bar{C}<1 \times 10^{-8} \mathrm{~kg} \mathrm{~m}^{-3}$.

Les résultats de la figure 1 correspondent à un cas stationnaire où la charge en matières en suspension dans la colonne d'eau s'adapte à chaque instant à la valeur du taux de remise en suspension $E$. On peut montrer que la concentration au fond se rattache à la valeur de $E$ par $C(0)=E / W$. De plus, quand la valeur de $U_{c}$ diminue, dès que $E=0$, les valeurs de $C(0)$ et de $\bar{C}$ deviennent très rapidement nulles après un laps de temps qui vaut environ $d / W$.

La figure 2 correspond à une période du signal $U_{c}$ qui vaut $T=600 \mathrm{~s}$. Les résultats du modèle $\alpha-\beta$ présentés sur la figure 2(a) montrent d'une part, que le signal du paramètre $\alpha$ est amorti et en retard sur celui du paramètre $\alpha_{\infty}$. D'autre part, le paramètre $\beta$ prend des valeurs négatives au début des processus de remise en suspension et des valeurs positives à la fin du processus de dépôt. Ces résultats correspondent à un régime non stationnaire avec un faible effet des phénomènes de dépôt et d'érosion sur le profil vertical de la concentration qui se manifeste par des valeurs de $\beta$ proches de zéro.

Les figures $2(b)$ et $2(c)$ confirment une très bonne qualité des résultats obtenus avec le modèle $\alpha-\beta$ dans ce cas étudié. Dans les applications pratiques le profil vertical de la concentration pourrait être ici correctement décrit par l'équation 6 avec $\alpha$ régi par l'équation 7 et $\beta$ fixé à zéro.

\subsection{Résultats pour les faibles périodes $(2,5 \leq T(\mathrm{~s}) \leq 10)$}

Les résultats présentés sur les figures 3 et 4 sont associés à une profondeur $d=2 \mathrm{~m}$ et à une période $T=10 \mathrm{~s}$. La figure 3 correspond à un diamètre $D=10 \mathrm{~mm}\left(W=0,690 \mathrm{~m} \mathrm{~s}^{-1}\right)$ et la figure 4 à un diamètre $D=0,2 \mathrm{~mm}\left(W=0,0264 \mathrm{~m} \mathrm{~s}^{-1}\right)$. Dans les deux cas les résultats du modèle $\alpha-\beta$, (figures $3(a)$ et $4(a)$ ), montrent que le signal du paramètre $\alpha$ est très amorti et très en retard sur celui du paramètre $\alpha_{\infty}$. Le paramètre $\beta$ reste toujours positif, ce qui se traduit par une réduction de la valeur de $C(0)$ par rapport à celle correspondant au profil de $C$ décrit par l'équation 6 (effet caractéristique du dépôt).

Même si des écarts sont observés entre les valeurs de $C(0) / \bar{C}$ issus des modèles $1 \mathrm{DV}$ et $\alpha-\beta$, les résultats obtenus pour $\bar{C}$ avec le modèle $\alpha-\beta$ restent satisfaisants. 


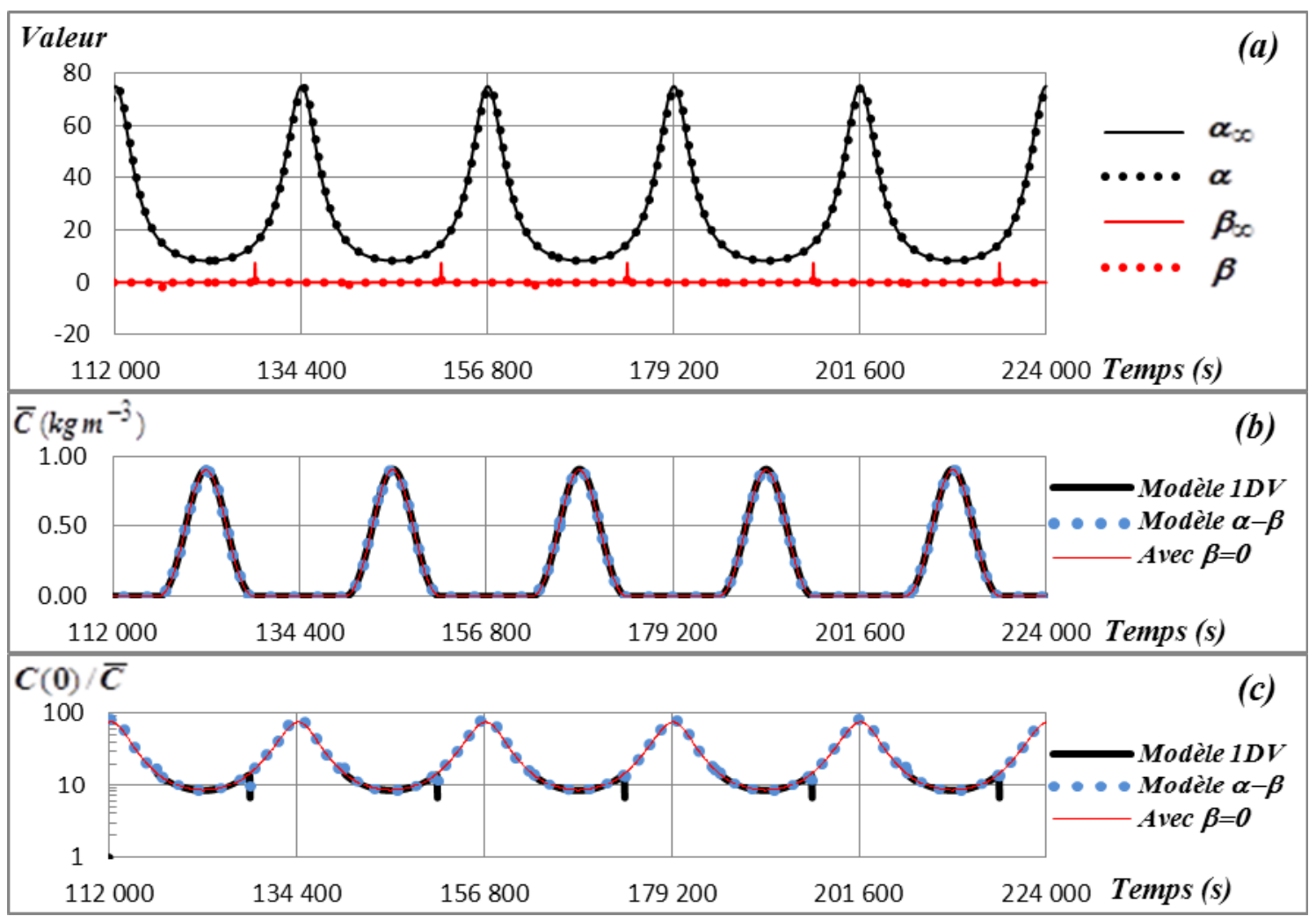

Figure 1. Résultats des modélisations avec $d=2 \mathrm{~m}, T=22400 \mathrm{~s}$ et $D=0,5 \mathrm{~mm}$.

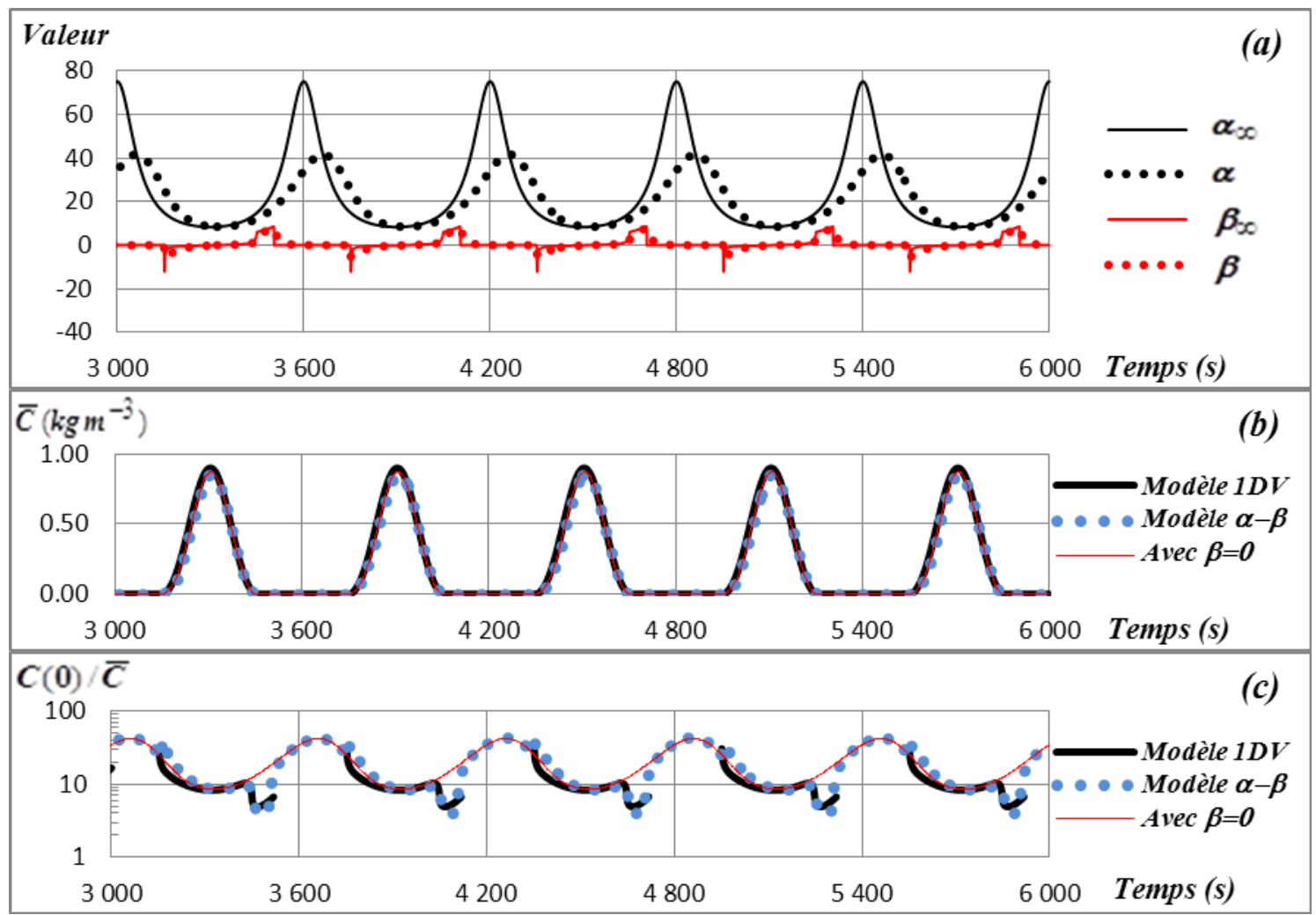

Figure 2. Résultats des modélisations avec $d=2 \mathrm{~m}, T=600 \mathrm{~s}$ et $D=0,5 \mathrm{~mm}$. 
Thème 2 - Dynamique sédimentaire

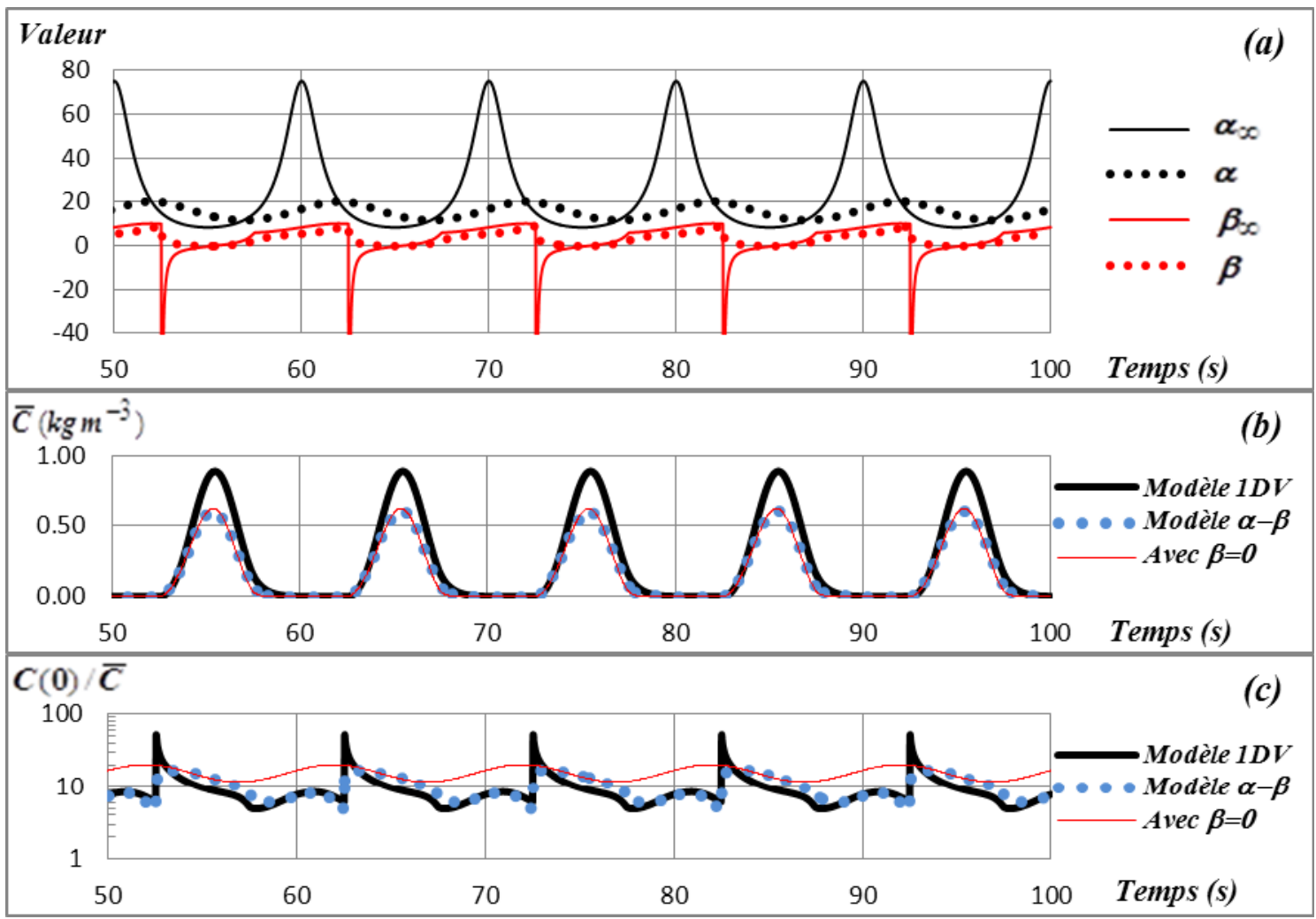

Figure 3. Résultats des modélisations avec $d=2 \mathrm{~m}, T=10 \mathrm{~s}$ et $D=10 \mathrm{~mm}$.

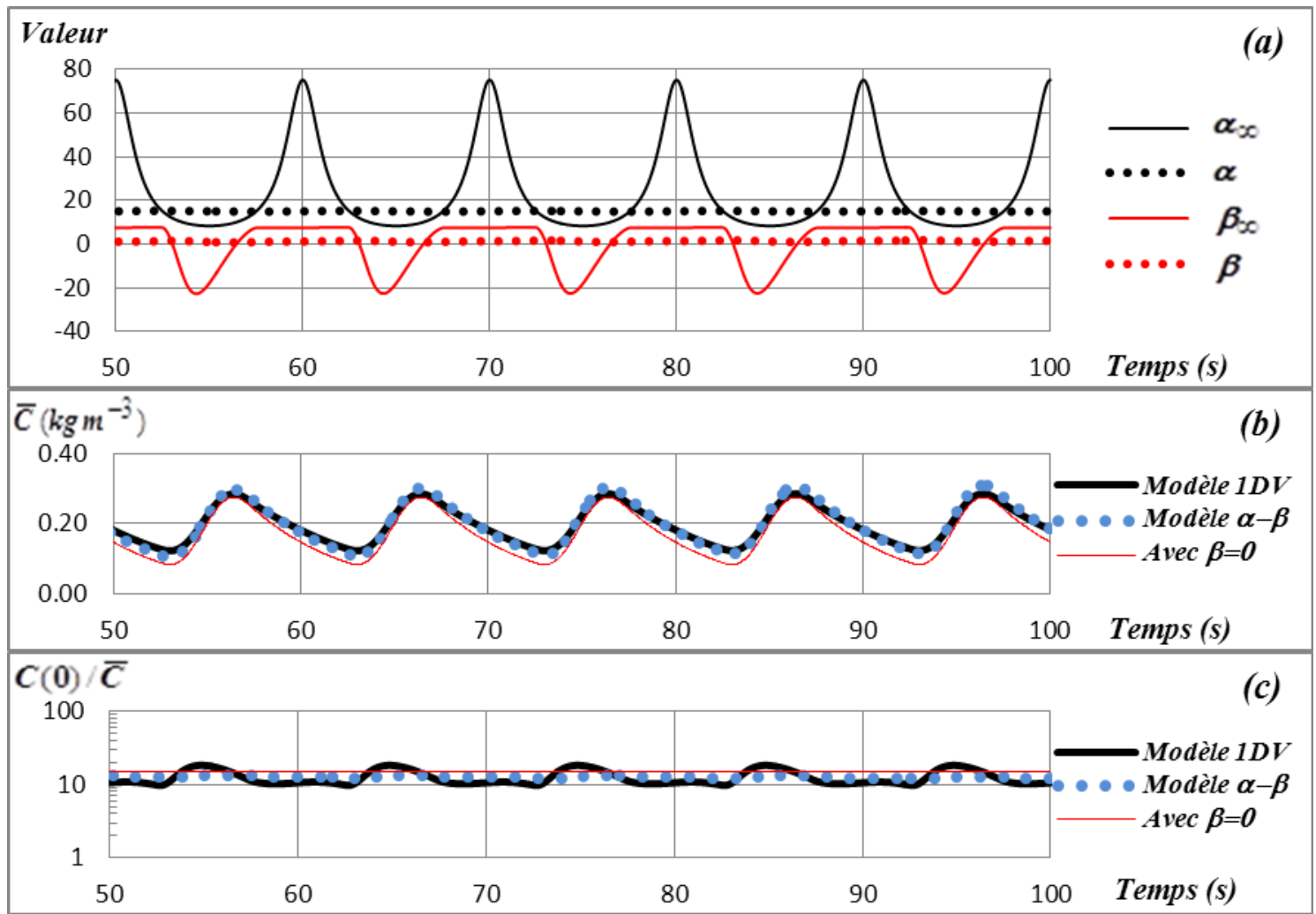

Figure 4. Résultats des modélisations avec $d=2 \mathrm{~m}, T=10 \mathrm{~s}$ et $D=0,2 \mathrm{~mm}$. 
On doit noter que dans les deux cas étudiés sur les figures 3 et 4 , la valeur de $\bar{C}$ issue du modèle $1 \mathrm{DV}$ est toujours supérieure à $1 \times 10^{-8} \mathrm{~kg} \mathrm{~m}^{-3}$ ce qui permet le calcul des valeurs théoriques du rapport $C(0) / \bar{C}$ pendant tout le cycle de variation de $U_{c}$.

Les résultats présentés sur les figures 5 correspondent à une profondeur $d=2 \mathrm{~m}$, à une période $T=2,5 \mathrm{~s}$ et à un diamètre des grains de sédiment $D=0,5 \mathrm{~mm}\left(W=0,0723 \mathrm{~m} \mathrm{~s}^{-1}\right)$. Les résultats du modèle $\alpha-\beta$ donnent des valeurs du paramètre $\alpha$ complétement amorties et presque constantes $\alpha \approx 15$. Les valeurs de $\beta \approx 1,10$ restent également presque invariantes dans le temps. Malgré l'écart existant entre les valeurs de $C(0) / \bar{C}$ propres aux modèles $1 \mathrm{DV}$ et $\alpha-\beta$, les résultats obtenus pour $\bar{C}$ avec le modèle $\alpha-\beta$ sont très précis dans ce cas étudié. On constate que les résultats restent satisfaisants quand la valeur du paramètre $\beta$ est bloquée à zéro.

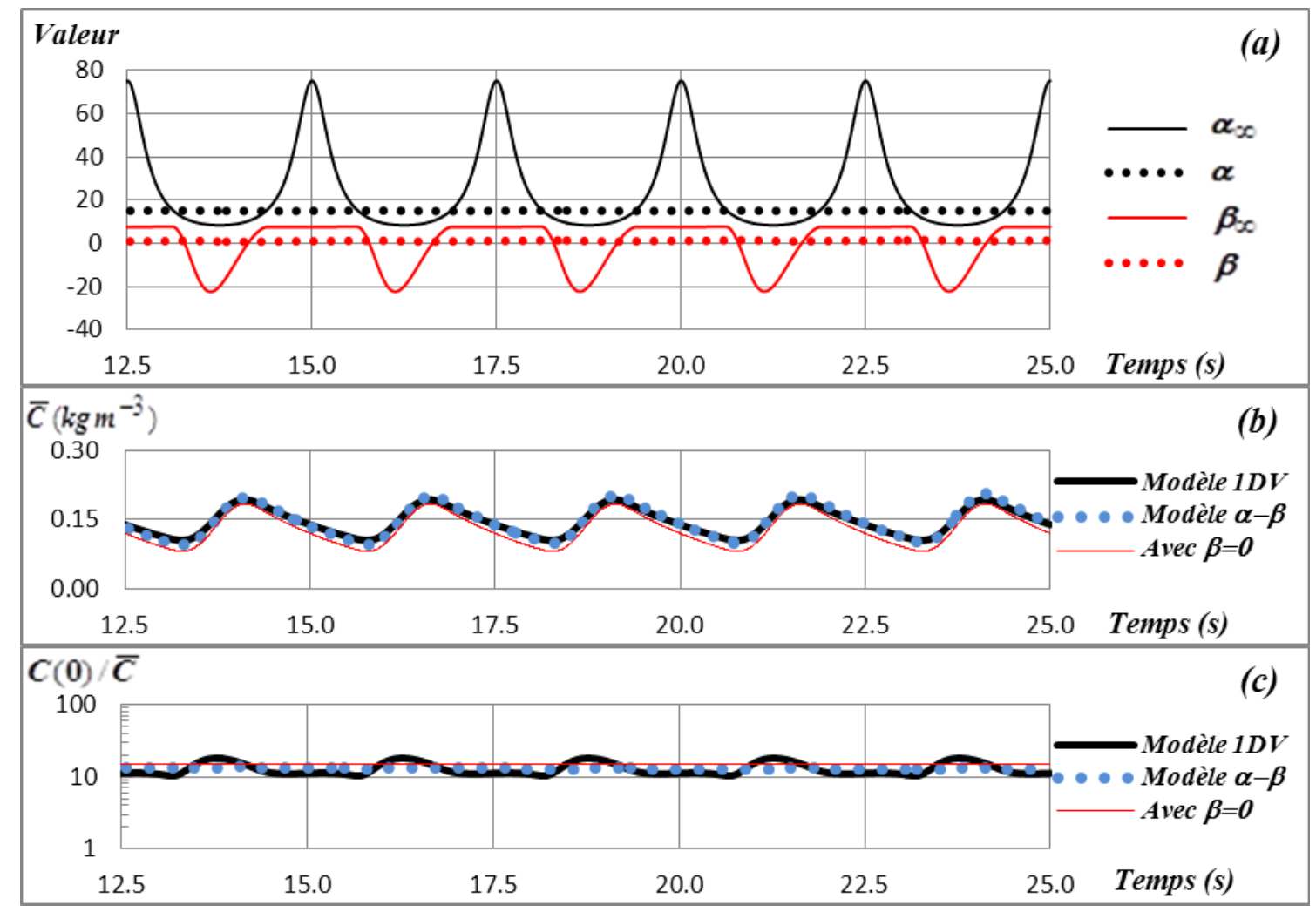

Figure 5. Résultats des modélisations avec $d=2 \mathrm{~m}, T=2,5 \mathrm{~s}$ et $D=0,5 \mathrm{~mm}$.

\section{Discussion}

Les travaux de TEETER (1986) ont montré qu'une bonne connaissance du rapport $C(0) / \bar{C}$ pendant les phases de dépôt est primordiale pour obtenir de bons résultats dans les modélisations $2 \mathrm{DH}$ du transport des sédiments fins en suspension. Cette étude montre à son tour que dans le cas des sédiments granulaires dont le taux de remise en suspension est décrit par une fonction "pick-up" la connaissance du rapport $C(0) / \bar{C}$ est nécessaire aussi pendant les phases d'érosion pour un bon calcul des échanges solides avec le fond (voir équation 16). 
Les coefficients $c_{\alpha}$ et $c_{\beta}$ du modèle $\alpha-\beta$ ont été initialement évalués pour des sédiments fins avec des vitesses de chute $W$ faibles $\left(0,05<W\left(\mathrm{~mm} \mathrm{~s}^{-1}\right)<3,20\right)$ et avec des variations des forçages hydrodynamiques correspondant à des périodes propres aux marées semidiurnes. Les résultats obtenus avec ce même modèle pour la distribution de sables et de graviers en suspension dans une colonne d'eau, sont satisfaisants dans tous les cas étudiés ici sans modification spécifique des coefficients $c_{\alpha}$ et $c_{\beta}$. La gamme des vitesses de chute étudiées est très large (voir tableau 1) pour des forçages hydrodynamiques cycliques couvrant toute la gamme des périodes observable en milieu côtier $(T>2,5 \mathrm{~s})$.

Dans les travaux de SANCHEZ (2013) le coefficient $c_{\beta}$ est rattaché à $\beta$ sur la base des résultats de simulations numériques effectuées avec $\beta=\beta_{\infty}$. Cette étude montre que quand $\beta \neq \beta_{\infty}$ les résultats s'améliorent si le coefficient $c_{\beta}$ est rattaché à $\beta_{\infty}$ à travers l'équation 13 .

\section{Conclusion}

La distribution verticale des matières en suspension est très bien décrite par le modèle $\alpha-\beta$ dans les cas de transport de sables et de graviers. Avec des forçages cycliques de période relativement longue on obtient $\alpha \approx \alpha_{\infty}$ (figure 1). Quand cette période diminue le signal du paramètre $\alpha$ est amorti et en retard sur celui du paramètre $\alpha_{\infty}$ (figures 2,3 et 4). Enfin au-dessous d'une certaine période très courte le signal du paramètre $\alpha$ est complétement amorti et presque invariant dans le temps (figure 5).

Bien que la prise en compte du paramètre $\beta \neq 0$ permette dans tous les cas une amélioration des résultats, ceux-ci restent satisfaisants quand on fixe $\beta=0$. Cela signifie que l'effet de l'érosion et du dépôt sur le profil vertical de la concentration dans le cas des sables et des graviers est peu significatif.

\section{Références bibliographiques}

SANCHEZ M. (2013). Description non stationnaire de la distribution verticale des sédiments transportés en suspension par les écoulements à surface libre, en présence de dépôt et d'érosion. Revue Paralia, Vol. 6, pp 9.1-9.30. http://dx.doi.org/10.5150/revueparalia.2013.009

SANCHEZ M., GRIMIGNI P., DELANOË Y. (2005). Steady-state vertical distribution of cohesive sediments in a flow. Comptes Rendus Geoscience, Vol. 337, pp 357-365. http://dx.doi.org/10.1016/j.crte.2004.10.020

TEETER A.M. (1986). Vertical transport in fine-grained suspension and newlydeposited sediment. In A.J. Mehta (Ed.), Estuarine cohesive sediment dynamics, Coastal and estuarine studies n ${ }^{\circ}$ 14, Springer, Berlin, pp. 170-191. http://dx.doi.org/10.1007/978-1-46124936-8 9

VAN RIJN L.C. (1984). Sediment pick-up functions. Journal of Hydraulic Engineering, Vol. 110(10), pp 1494-1502. http://dx.doi.org/10.1061/(ASCE)0733-9429(1984)110:10(1494) 